\title{
Containing 2019-nCoV (Wuhan) coronavirus
}

\author{
Edward H. Kaplan ${ }^{1}$ (1)
}

Received: 6 February 2020 / Accepted: 12 February 2020 / Published online: 7 March 2020

(C) Springer Science+Business Media, LLC, part of Springer Nature 2020

\begin{abstract}
The novel coronavirus 2019-nCoV first appeared in December 2019 in Wuhan, China. While most of the initial cases were linked to the Huanan Seafood Wholesale Market, person-to-person transmission has been verified. Given that a vaccine cannot be developed and deployed for at least a year, preventing further transmission relies upon standard principles of containment, two of which are the isolation of known cases and the quarantine of persons believed at high risk of exposure. This note presents probability models for assessing the effectiveness of case isolation and quarantine within a community during the initial phase of an outbreak with illustrations based on early observations from Wuhan.
\end{abstract}

Keywords Coronavirus $\cdot$ Containment $\cdot$ Isolation $\cdot$ Quarantine $\cdot$ Probability modeling

The novel coronavirus 2019-nCoV first appeared in December 2019 in Wuhan, China [1]. Most of the initial cases were linked to the Huanan Seafood Wholesale Market, but person-to-person transmission was established quickly while viral transmission prior to the appearance of symptoms remains controversial $[2,3]$. From the same family as the SARS and MERS coronaviruses $(10 \%$ and $35 \%$ fatality rates respectively $[4,5]), 2019-\mathrm{nCoV}$ has also led to serious cases of pneumonia, albeit with a lower estimated fatality rate of $2-3 \%$ at the present time [6]. Given that a vaccine cannot be developed and deployed for at least a year, preventing further transmission relies upon standard principles of containment, two of which are the isolation of known cases and the quarantine of persons believed at high risk of exposure (with the latter extended inside China to prevent travel to or from Wuhan, and globally via the cancellation of air travel to and from China).

What follows are some probability models for assessing the effectiveness of case isolation of infected individuals and quarantine of exposed individuals within a community during the initial phase of an outbreak with illustrations based on early observations from Wuhan. The good news is that in principle, case isolation alone is sufficient to end

Edward H. Kaplan

edward.kaplan@yale.edu

1 William N. and Marie A. Beach Professor of Operations Research, Professor of Public Health, Professor of Engineering, Yale School of Management, 165 Whitney Avenue, New Haven 06511, CT, USA community outbreaks of 2019-nCoV transmission provided that cases are detected efficiently. Quarantining persons identified via tracing backwards from known cases is also beneficial, but less efficient than isolation.

To begin, suppose someone has just become infected. Absent intervention, assume that this infected person will transmit new infections in accord with a time-varying Poisson process with intensity function $\lambda(t)$ denoting the transmission rate at time $t$ following infection. The expected total number of infections this person will transmit over all time (the reproductive number $R_{0}$ ) equals

$R_{0}=\int_{0}^{\infty} \lambda(u) d u$

and as is well-known, an epidemic cannot be self-sustaining unless $R_{0}>1$ [7, 8]. It follows that a good way to assess isolation and quarantine is to examine their effect on $R_{0}$. But first, we take advantage of another epidemic principle, which is that early in an outbreak, the incidence of infection grows exponentially. So, suppose that the rate of new infections grows as $k e^{r t}$ where $r$ is the exponential growth rate, and let $\iota_{0}$ denote the initial number of infections introduced at time 0 . It follows that

$k e^{r t}=\int_{0}^{t} k e^{r(t-u)} \lambda(u) d u+\iota_{0} \lambda(t), \quad t>0$

which is to say that the rate of new infections at chronological time $t$ is the cumulation of all past infections times the chronological time $t$ transmission rate associated with those past infections. Simplifying and recognizing that 
$e^{-r t} \lambda(t)$ goes to zero ( $R_{0}$ is finite) yields the Euler-Lotka equation

$\int_{0}^{\infty} e^{-r u} \lambda(u) d u=1$.

In the disease outbreak context, Eq. 3 can be understood as the composite of all sources of current infections. Among all persons newly infected, the fraction whose infectors were infected between $t$ and $t+\Delta t$ time units ago equals $e^{-r t} \lambda(t) \Delta t$. The function

$b(t)=e^{-r t} \lambda(t), t>0$

is thus the probability density for the duration of time an infector has been infected as sampled from the infectors of those just infected.

Back to Wuhan, where detailed study of the first 425 confirmed 2019-nCoV cases was reported in [1]. Using only case data up to January 4, the exponential growth rate $r$ was directly estimated to equal $0.1 /$ day [1]. Contact tracing from identified index cases was able to establish links to their presumed infectors. While it was not possible to pinpoint exact dates of infection, the dates at which symptoms in both infectees and (presumed) infectors occurred were determined, and the difference in these dates taken as a proxy for the elapsed time since infection of the infector (see [7] for technical issues that arise from this approach). The resulting frequency distribution was then used to estimate $b(t)$, which was fit as a gamma distribution with mean (standard deviation) of 7.5 (3.4) days [1]. Given these estimates of $r$ and $b(t), \lambda(t)=e^{r t} b(t)$ and

$R_{0}=\int_{0}^{\infty} e^{r u} b(u) d u=2.26$,

consistent with what was reported in [1] as well as other studies employing different methods $[9,10]$.

We can now model containment. Starting with case isolation, suppose that an infected person is detected at time $T_{D}$ days following infection, and is isolated for $\tau_{I}$ days. The effect of doing this is to erase all infections that would have been transmitted between times $T_{D}$ and $T_{D}+\tau_{I}$. Following the Poisson model, the expected number of transmissions blocked equals $\int_{T_{D}+\tau_{I}}^{T_{1}} \lambda(u) d u$. Clearly the sooner an infected person is detected (the smaller $T_{D}$ ) and the longer a person is isolated (the larger $\tau_{I}$ ), the greater the number of infections that can be prevented. Suppose that newly infected persons self-recognize their infection at the time when symptoms appear. This optimistic scenario equates the detection time to the incubation time for 2019$\mathrm{nCoV}$, and this incubation time distribution was reported to follow a lognormal distribution with a mean of 5.2 days and a 95th percentile of 12.5 days (which implies a standard deviation of 3.9 days) [1]. Denoting the incubation time density by $f_{T_{D}}(t)$, the expected number of transmissions blocked by case isolation of duration $\tau_{I}$ upon the appearance of symptoms, $\beta_{I}$, is given by

$\beta_{I}=\int_{0}^{\infty} f_{T_{D}}(x) \int_{x}^{x+\tau_{I}} \lambda(u) d u d x$

Substituting $\lambda(t)$ and $f_{T_{D}}(t)$ as previously described yields $\beta_{I}$ 's of $1.22,1.71$ and 1.78 for 7,14 and unlimited days in isolation. The best one can hope for from case isolation is that 1.78 of 2.26 transmissions would be blocked, which would reduce the effective reproductive number to $2.26-$ $1.78=0.48$, well below threshold. Isolation of only one week almost reduces transmission to one new infection per case.

However, assuming that the time to detection is equal to the incubation time is very optimistic. Indeed, the Wuhan study revealed that the average time from onset of illness to a medical visit was 5.8 days [1], comparable to the incubation time. To obtain a more sobering view of isolation, suppose that an individual's time to detection is twice the incubation time. Using the lognormal incubation density cited above, the new detection time distribution will also be lognormal but now with a mean (standard deviation) of 10.4 (7.8) days. Applying Eq. 6 yields $\beta_{I}$ 's of $0.84,1.07$ and 1.1 for isolations of 7, 14 and unlimited days. Even lifetime isolation fails to reduce transmission below threshold if the time to detection takes too long. Given the amount of attention generated by news coverage and public service announcements, this second scenario is overly pessimistic. The real message is the importance of rapid (self) detection.

What of quarantine? Screening and quarantining individuals potentially exposed elsewhere upon entry to a community (as has been the case at airports) certainly can prevent the importation of new infections and their subsequent transmission chains, though at the cost of containing uninfected persons. Beyond this, quarantine (typically at home where it is recommended that the exposed person not share immediate space, utensils, towels etc. with others) is meant for apparently healthy individuals discovered to be at risk of exposure via contact tracing with the idea that should they in fact have become infected, they would become ill without transmitting the virus and then report for isolation. However, quarantining uninfected contacts offers no benefit presuming the potential infector has already been identified and isolated, so the key question is whether such tracing would reach already infected but previously unidentified contacts in time to make a meaningful reduction in disease transmission.

To present an optimistic view of tracing-driven quarantine, suppose that a newly infected person (referred to as the index from the standpoint of contact tracing) is immediately identified. Instantaneous interview and tracing leads to the 
quarantine of our index's prior contacts, one of whom happens to be the infector (who is immediately isolated upon discovery). Said infector, however, has already been infectious for some time before being identified via the index case. Indeed, the probability density for the duration of time the infector has already been infected is given by Eq. 4 . Suppose that the infector is placed in quarantine for $\tau_{Q}$ days. The expected number of transmissions that would be blocked, $\beta_{Q}$, is given by

$\beta_{Q}=\int_{0}^{\infty} b(x) \int_{x}^{x+\tau_{Q}} \lambda(u) d u d x$.

While the equations for $\beta_{I}$ and $\beta_{Q}$ have the same structure, there is a key difference. The elapsed time from infection until an infected person enters isolation directly depends upon the time to recognize symptoms, which is related fundamentally to the incubation time distribution. The elapsed time from infection until an infected person enters quarantine/isolation via contact tracing, however, depends upon sampling from those newly infected and looking backwards to estimate the infector's elapsed duration of infection.

Using the previously estimated models for $b(t)$ and $\lambda(t)$, Eq. 7 yields $\beta_{Q}$ 's of $1.05,1.33$ and 1.36 for $\tau_{Q}$ 's of 7,14 , and unlimited days. The 14 day quarantine proposed in [1] would reduce the effective reproductive number to $2.26-$ $1.33=0.93$, which is just under threshold. Again, this is an optimistic view of contact tracing, for identification of the infector is presumed instantaneous at the index's time of infection. Taking into account the detection delay in recognizing the index case would similarly delay the identification of the infector via contact tracing, reducing the number of transmissions that could be prevented as a result.

There is no either/or choice between quarantine and isolation. Using both leads to an infected person being detected at the minimum of the time a person selfdetects due to symptoms and the time a person would be identified via contact tracing. The expected number of infections prevented then follows from Eq. 6 after substituting the probability density for the minimum of the two detection times. To illustrate, assume independence between self-identification and contact-tracing detection times, that self-identification occurs at twice the incubation time, contact identification times follow $b(t)$ as previously, and quarantine/isolation is unlimited in duration. The associated $\beta_{I Q}$ denoting expected infections averted via isolation and quarantine now equals 1.64 , which reduces the reproductive number from 2.26 to 0.62 , well below the epidemic threshold.

The preceding analysis has focused on reducing the reproductive number below 1 , yet doing so can still lead to a large total number of infections. For example, reducing the reproductive number to 0.9 would lead to ten times as many infections in total as the extant number at the start of containment, as total infections in such a "minor" outbreak scales as $1 /\left(1-R_{0}\right)[11]$.

The modeling above is meant to be illustrative and surely could be improved in many ways. Appropriate characterization of underlying statistical uncertainty, better operational modeling of how actual isolation, quarantine and contact tracing operate [12] (including voluntary selfquarantine by untraced persons who might have been exposed), consideration of the costs of intervention as well as the public health benefits, and characterizing the appropriate level of resources to devote to this outbreak relative to other arguably more pressing public health concerns are all subjects deserving careful study.

Additional common-sense precautions such as regular handwashing, the use of facemasks, and other measures not considered here should help make such outbreaks even more manageable. One important suggestion is that people should receive flu shots, for in addition to protecting against influenza, vaccination would reduce the number of false positive 2019-nCoV cases reported since fewer people would have the common symptoms of both flu and coronavirus, and if a vaccinated person did get sick, it would raise the probability that the case is coronavirus as opposed to flu and make it more likely said person would seek care [13]. There are other practical aspects to explore, including the development of a less-precise but more rapid diagnostic mechanism, determining how long one can safely delay ill patients with symptoms from coming to the hospital to help alleviate congestion, and figuring out how quickly Airborne Infection Isolation Rooms (negative pressure units) can be created by hacking the ventilation system in ordinary wards to increase isolation capacity [14].

Nonetheless, the modeling results obtained are reassuring. Containment via isolation and quarantine has the capacity to control a community 2019-nCoV outbreak.

Acknowledgments I thank Ron Brookmeyer, Forrest Crawford, Gregg Gonsalves, Robert Heimer, Albert Ko, Barry Nalebuff, David Paltiel, Greg Zaric and an anonymous referee for comments; any errors are my own.

\section{References}

1. Li Q, Guan X, Wu P et al (2020) Early transmission dynamics in Wuhan, China, of novel coronavirus-infected pneumonia. N Engl J Med. https://doi.org/10.1056/NEJMoa2001316

2. Rothe C, Schunk M, Sothmann P et al (2020) Transmission of 2019-nCoV infection from an asymptomatic contact in Germany. N Engl J Med. https://doi.org/10.1056/NEJMc2001468

3. Kupferschmidt K (2020) Study claiming new coronavirus can be transmitted by people without symptoms was flawed. Science February 3, 2020. https://www.sciencemag.org/news/2020/02/ 
paper-non-symptomatic-patient-transmitting-coronavirus-wrong. Accessed 5 February 2020

4. Chan-Yeung M, Xu R-H (2003) SARS: Epidemiology. Respirology 8:S9-S14. https://onlinelibrary.wiley.com/doi/epdf/10.1046/j. 1440-1843.2003.00518.x

5. Killerby ME, Biggs HM, Midgley CM, Gerber SI, Watson JT (2020) Middle East respiratory syndrome coronavirus transmission. Emerg Infect Dis 26(2):191-198. https://doi.org/10.3201/ eid2602.190697

6. Wang C, Horby PW, Hayden FG, Gao GF (2020) A novel coronavirus outbreak of global health concern. Lancet. https://doi. org/10.1016/S0140-6736(20)30185-9

7. Britton T, Tomba GS (2019) Estimation in emerging epidemics: Biases and remedies. J R Soc Interface 16:20180670. https://doi.org/10.1098/rsif.2018.0670

8. Anderson RM, May RM (1991) Infectious diseases of humans: Dynamics and control. Oxford University Press, Oxford

9. Wu JT, Leung K, Leung GM (2020) Nowcasting and forecasting the potential domestic and international spread of the 2019-nCoV outbreak originating in Wuhan, China: A modelling study. Lancet. https://doi.org/10.1016/S0140-6736(20)30260-9

10. Imai N, Cori A, Dorigatti I, Baguelin M, Donnelly CA, Riley S, Ferguson NM (2020) Report 3: Transmissibility of 2019-nCoV
MRC centre for global infectious disease analysis. Imperial College, London. https://www.imperial.ac.uk/media/imperial-college/ medicine/sph/ide/gida-fellowships/Imperial-2019-nCoV-transmissibility.pdf, Accessed 1 February 2020

11. Becker NG (2015) Modeling to inform infections disease control. CRC Press, Chapman \& Hall, New York

12. Kaplan EH, Craft DL, Wein LM (2003) Analyzing bioterror response logistics:the case of smallpox. Math Biosci 185:33-72. https://doi.org/10.1016/S0025-5564(03)00090-7

13. Borio L, Gottlieb S (2020) Act now to prevent an American epidemic: Quarantines, flu vaccines and other steps to take before the Wuhan virus becomes widespread. Wall Street Journal January 28, 2020. https://www.wsj.com/articles/act-now-to-preventan-american-epidemic-11580255335, Accessed 2 February 2020

14. Miller SL, Clements N, Elliott SA, Subhash SS, Eagan A, Radonovich LJ (2017) Implementing a negative-pressure isolation ward for a surge in airborne infectious patients. Am J Infection Control 45:652-659. https://www.ajicjournal.org/article/ S0196-6553(17)30092-5/pdf

Publisher's note Springer Nature remains neutral with regard to jurisdictional claims in published maps and institutional affiliations. 